\title{
Sweetness suppression in fructose/citric acid mixtures: A study of contextual effects
}

\author{
HENDRIK N. J. SCHIFFERSTEIN \\ Agricultural University, Wageningen, The Netherlands
}

\begin{abstract}
Two experiments investigated whether stimulus context affects ratings for mixtures of dissimilartasting substances (fructose/citric acid) to the same degree that it affects ratings for unmixed substances (fructose). In Experiment 1, replacing mixtures by equisweet unmixed fructose solutions produced virtually no response shifts. The proportion of mixtures in the stimulus set affected only slightly the degree of mixture suppression inferred from the responses. In Experiment 2, both the stimulus type (mixed or unmixed) and the stimulus distribution (positively versus negatively skewed) affected the responses. Several factors that determine the impact of contextual changes are identified: (a) the stage in stimulus processing affected-that of representation on the internal continuum or that of response selection; (b) the size and sources of variation in the affected process; and (c) the degree to which a stimulus is perceptually integrated in the context. In the present study, the sweetness of fructose/citric acid mixtures was largely, but not completely, integrated with the sweetness of unmixed fructose solutions. It is suggested that increased stimulus complexity makes mixture ratings more susceptible to contextual shifts. An analysis relating the size of the contextual shift to the degree of response variability suggests that response-selection processes are more important in determining the responses for unmixed stimuli than they are in determining the responses for mixtures.
\end{abstract}

Stimulus spacing, stimulus frequency, and stimulus range all affect the mean responses to stimuli. Mean intensity ratings are higher when many low-intensity stimuli are presented than when many high-intensity stimuli are presented (e.g., McBride, 1985; Riskey, 1982; Riskey, Parducci, \& Beauchamp, 1979; Schifferstein \& Frijters, 1992a). Several investigators have developed theoretical models to account for contextual effects in experiments employing stimuli that differed in perceived intensity only (e.g., Haubensak, 1992; Helson, 1964; Parducci, 1965, 1974; Parducci \& Wedell, 1986).

Intensity judgments for mixtures of dissimilar-tasting substances depend on stimulus context, too. In some studies, the characteristics of the preceding unmixed stimuli were found to affect intensity judgments for mixed and unmixed stimuli differentially (Kroeze, 1983). In other studies, this differential effect was not significant (Schifferstein, 1994b; Schifferstein \& Frijters, 1992b). Kroeze (1982) reported that an increase in the relative number of mixtures in the stimulus set increased the mixture responses.

I gratefully acknowledge the funding given to this project by the Netherlands Organization for Scientific Research (NWO PSYCHON Grant 560-262-032, awarded to J. E. R. Frijters). I wish to thank Jan Frijters, Larry Marks, and two anonymous reviewers for their comments on previous versions of this article, and Ralf Kleykers for collecting the data. Correspondence should be addressed to H. N. J. Schifferstein, Department of Marketing and Marketing Research, Agricultural University, Bomenweg 2, 6703 HD Wageningen, The Netherlands (e-mail: rick.schifferstein@alg.menm.wau.nl).
In mixtures of qualitatively different substances, the sensation elicited by one component is usually suppressed by the other component, a phenomenon called mixture suppression (e.g., Pangborn, 1960). A differential context effect on ratings for mixed and unmixed stimuli necessarily affects the degree of suppression inferred from the responses. Consequently, stimulus context may be a complicating, and hitherto neglected, factor relevant to mixture research.

\section{A Theoretical Framework for the Study of Contextual Effects}

According to a Stimulus-Organism-Response view of single stimulus intensity judgment, three different stages can be distinguished in stimulus processing (Figure 1). First, a psychophysical function transforms the physical stimulus into a subjective experience, a percept, regarded as the output from the sensory system. Mixture suppression is assumed to reflect the operation of peripheral and central processes of sensory interaction (e.g., Kroeze \& Bartoshuk, 1985). In addition, sensory adaptation results in a decrease in the summated chorda tympani response (e.g. Borg, Diamant, Oakley, Ström, \& Zotterman, 1967) and thus affects the first processing stage.

The percept, temporarily stored in the sensory buffer, is encoded to enable storage in memory and judgmental processing. During encoding, nontarget elements of a complex percept may influence the subject. Attentive processes enable the subject to focus upon the sensation attribute required by the experimental task. The subject's conception of what is meant by the requested attribute 


$\begin{array}{cccc}\text { Physical } & \text { Perceived } & \text { Coded } & \text { Overt } \\ \text { concentration } & \text { sensation sensation response }\end{array}$

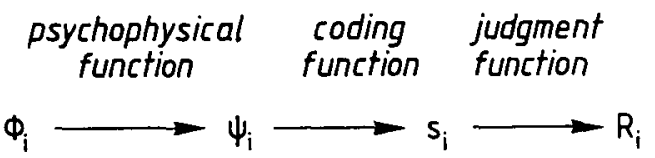

Figure 1. The pathway from physical stimulus to observed response, according to a Stimulus-Organism-Response view of single stimulus judgment.

(e.g., "sweetness intensity") affects stimulus processing at this stage. After encoding, the stimulus is represented on an internal, subjective continuum. The coded sensation is no longer a sensation, but is instead a cognition. Any sensory scale derived from rated intensities necessarily represents coded sensations (Frijters, 1993).

After encoding, the third processing stage transfers the values of the coded sensations on the subjective continuum into responses on the scale provided. This process reflects the judgment function or response-output function.

In principle, contextual shifts in responses could be the result of changes in any of the three stages of stimulus processing - the psychophysical stage, the coding stage, or the judgmental stage. Since contextual effects are probably not due to sensory mechanisms such as adaptation (Riskey, 1982), it seems reasonable to assume that the psychophysical stage is unaffected by experimental context. ${ }^{1}$ Hence, contextual effects probably result from changes in encoding or judgment functions.

To understand the mechanism of contextual changes in stimulus processing, the following three factors seem important: (a) the type of process affected by contextual changes; (b) the size of the variation and the sources of variation in the affected process; and (c) the degree to which the subjects regard the experimental stimulus as part of the manipulated context.

\section{Variation During Encoding}

Stimulus processing probably requires more cognitive effort for a heterogeneous percept than it does for a homogeneous one. For example, judging the sweetness intensity of a sucrose/ $\mathrm{NaCl}$ mixture appears more demanding than does judging the sweetness of unmixed sucrose. When the mixture is evaluated, one sensory quality has to be ignored while the other is judged; the subjects have to decide which part of the subjective experience corresponds to "sweetness" and which part to "saltiness." The presence of irrelevant taste sensations may interfere during classification and encoding of sensory input, resulting in a fuzzy representation on the requested sensory continuum. The more ambiguous the stimulus information, the more the subject will use nonrelevant cues in selecting a response. For example, Ward (1979) has shown that the present response is more and more determined by the previous response if the fuzziness of the internal representations increases.

Besides adding noise to responses, the fuzziness in the internal representation might make judgments more susceptible to systematic variation. Consequently, increased perceptual complexity could increase both the standard deviation of the mean rating and the size of any contextual shift. Thus, if mixtures differ from unmixed stimuli in physical and perceptual complexity, this distinction could be the source of a differential context effect. Note, however, that no one-to-one relationship exists between the number of physical components of a mixture and its perceived complexity (Erickson, 1982; Erickson \& Covey, 1980): The degree of complexity of a mixture percept varies with the number of physical components, and with the ratio and the absolute concentration levels of the physical components present (O'Mahony, Atassi-Sheldon, Rothman, \& MurphyEllison, 1983).

\section{Variation in Response Selection}

In the response-selection process, the degree of variation is likely to be determined by the number of feasible response alternatives. A larger number of response alternatives is likely to result in a larger degree of response variability. If responses are collected on category rating scales or line scales, the subjects are restricted in their range of possible responses. For category rating scales, the standard error of the mean rating is relatively small at either end of the response scale and larger in the middle of the scale (e.g., Marks, 1982; Montgomery, 1982). In selecting a momentary response, subjects may use nonrelevant, situation-dependent cues emerging from the experimental context, producing a positive association between degree of response variability and size of the contextual effect.

\section{Similarity}

The third factor affecting the size of a contextual effect is the degree to which a stimulus is integrated in the stimulus context. Whether a subject regards a stimulus as part of a certain context likely depends on the perceptual similarity between stimulus and context.

Similarity is generally minimal when stimuli are delivered to different modalities. When subjects judge overall intensity of a multimodal stimulus set on one response scale (e.g., Stevens \& Marks, 1980), judgments for the different modalities may be independent: two different psychophysical functions, two coding functions, and two response-output functions operate (Figure 1). According to Marks and Warner (1991), different channels of attention process the two types of stimuli in this case.

Independence from stimulus frequency distribution has been reported for several stimulus sets eliciting different qualities within one modality: Riskey and Desor (1980) showed that saltiness judgments for tomato juice were made independently of manipulations of the sweetness of cherry-flavored soft drinks, and vice versa. Par- 
ducci, Knobel, and Thomas (1976) showed that the size of circles can be judged independently from the distribution of simultaneously presented squares. However, the judgments of circle size were not entirely independent from the range in square sizes.

If both stimulus sets are perceived and processed independently, contextual manipulations of one stimulus set affect only the ratings for the "own" stimulus set and do not affect those for the "other" set. Consequently, comparing ratings over sets is not meaningful: Matching values vary with stimulus context, the so-called slippery context effect (Marks \& Warner, 1991). The relative, context-dependent component in judgment makes it possible for $1.0 \mathrm{M}$ sucrose to be equi-intense to $0.6 \mathrm{M}$ $\mathrm{NaCl}$ in one experiment (De Graaf \& Frijters, 1987) and equi-intense to $0.8 \mathrm{M} \mathrm{NaCl}$ in another experiment (De Graaf \& Frijters, 1989). Since the Weber fraction for $\mathrm{NaCl}$ at this concentration level is approximately $15 \%$ (Schutz \& Pilgrim, 1957), these two concentration levels are easily discriminated in a paired comparison.

When two stimulus sets are highly similar, the two stimulus sets combine into one generalized context. This type of interdependency usually occurs only for withinquality judgments. If the stimulus sets differ physically (e.g., different sweeteners are used to construct the two stimulus sets), different psychophysical functions may operate. However, encoding and judgment must be identical for the two stimulus sets (one process channel). If generalization occurs, the ratings for both stimulus sets are similarly affected by manipulations of either stimulus set. Stimulus matches do not vary with contextual changes, implying no slippery context effect. Generalization was reported by Vollmecke (1987), who had subjects rate the sweetness intensities of papaya and apricot beverages varying in sucrose concentration.

If the stimulus sets are neither fully integrated nor completely independent, the stimulus sets may be called dependent. This case falls somewhere between the generalized and the independent stimulus contexts. Two dependent stimulus sets are not perceptually similar enough to produce generalization, but they are not dissimilar enough to be processed independently. The size of the slippery context effect in the stimulus matches is smaller for dependent than for independent contexts, but is not absent as in a generalized context. The size of the slippery context effect decreases monotonically with the degree of qualitative similarity (Marks \& Warner, 1991; Rankin \& Marks, 1991). Also, the ratings for one stimulus set are affected by variations in the other set. The shifts in ratings are expected to be smaller for dependent sets than for generalized stimulus sets. Consequently, mean responses will be more affected by contextual changes in the "own" stimulus set than they will be by changes in the "other" stimulus set.

Table 1 shows an overview of the effects of a contextual shift in one stimulus set upon the ratings and the stimulus matches of the other set for independent, dependent, and generalized contexts.
Table 1

Effect of Shift in Stimulus Distribution of One Stimulus Set

Upon the Stimulus Matches and the Ratings for the Other Stimulus Set in Different Processing Contexts

\begin{tabular}{lcc}
$\begin{array}{c}\text { Contextual } \\
\text { Processing }\end{array}$ & Matches & Ratings \\
\hline Independence & ++ & - \\
Dependence & + & + \\
Generalization & - & ++
\end{tabular}

Note-No effect $=-$; Detectable effect $=+$; Substantial effect (larger than +$)=++$.

Summarizing, I hypothesize that experimental context affects stimulus processing if some internal coding or judgment process is subject to variation. Contextual manipulations will change the response to an experimental stimulus only if the stimulus is (to some degree) regarded as a part of this context.

\section{The Present Study}

The main objective of the present study was to find out whether contextual effects affect responses to mixed and unmixed stimuli to the same degree. In other words, the question of whether mixtures and unmixed stimuli are psychologically integrated (generalized) or separated (independent) during stimulus processing was investigated. The contextual variables investigated were the stimulus frequency distributions (Experiments 1 and 2) and the relative number of mixtures in the stimulus set (Experiment 1). Post hoc analyses investigated whether the contextual effects are larger for complex stimuli than they are for simple stimuli, and whether the size of the context effect is related to variation in responses. The aim of these analyses was to substantiate the theoretical framework presented above.

\section{EXPERIMENT 1}

The first experiment investigated whether equisweet skewed stimulus sets of fructose solutions and fructose/citric acid mixtures-varying in the percentage of mixtures - affect sweetness ratings for mixed and unmixed stimuli to the same degree.

\section{Method}

Subjects. Ninety-six paid volunteers, 28 men and 68 women, ranging in age from 17 to 51 years (median age 22), participated in the experiment. Most subjects were students of the Agricultural University and had little or no experience with psychophysical scaling tasks. They were naive with respect to the substances used and the purpose of the study.

Stimuli. The stimuli were solutions of fructose (Merck 5321) and mixtures of fructose with citric acid (Merck 244) in demineralized water. The nine fructose concentrations ranged from 0.125 to $2.0 \mathrm{M}$ in equal log steps. The eight fructose/citric acid mixtures formed two equiratio-mixture series of four solutions each (Frijters \& Oude Ophuis, 1983); the first mixture type is called FruCitr.99/.01, the second, FruCitr.98/.02. Equiratio mixtures have different total concentrations but a constant ratio of concentrations of constituent components (in molarity). For example, in the first mixture series (FruCitr.99/.01) the concentration ratio for the 
highest concentration level, a mixture of $2.0 \mathrm{M}$ fructose and $20 \mathrm{mM}$ citric acid, equals the concentration ratio in the second highest level, a mixture of $0.707 \mathrm{M}$ fructose and $7.07 \mathrm{mM}$ citric acid, whereas the solutions differ with respect to their total concentrations (i.e., $2.02 \mathrm{M}$ and $0.71407 \mathrm{M}$, respectively). The fructose concentrations in both mixture series were $0.177,0.354$, 0.707 , and $2.0 \mathrm{M}$. The citric acid concentrations in the FruCitr.99/ .01 mixture series were $1.8,3.5,7.1$, and $20 \mathrm{mM}$, respectively. For the FruCitr. $98 / .02$ series, they were $3.5,7.1,14.1$, and $40 \mathrm{mM}$, respectively. In addition to the fructose solutions and fructose/citric acid mixtures, water was used as an experimental stimulus. For reasons of standardization, the subjects were presented with two references, the first demineralized water, the second a solution of $2.25 \mathrm{M}$ fructose. Solutions were prepared at least $24 \mathrm{~h}$ before tasting and were stored in a dark, refrigerated room at $4^{\circ} \mathrm{C}$ for no longer than six days.

Design. The eight fructose/citric acid mixtures were used to obtain two different stimulus distributions. Pilot studies showed that an increase in citric acid level at a constant fructose level usually resulted in a decrease in sweetness rating. For neither of the citric acid levels did the sweetness ratings for one fructose level drop below the sweetness ratings for the next lower fructose level. Therefore, a mixture series increasing monotonically in sweetness intensity could be constructed by alternating between the two equiratio mixture types. This mixture series was then used to construct positively and negatively skewed stimulus distributions (Table 2, distributions PosMix and NegMix). The nine unmixed fructose solutions and one water sample were included in these skewed stimulus distributions to obtain a total of 30 samples (20 mixtures, 9 fructose, and 1 water) for each distribution.

To investigate whether context-dependent response shifts are determined by only the judged attribute (sweetness) or also by other attributes (sourness, total intensity) of the mixtures in the skewed distribution, four other stimulus distributions were constructed. These were hypothesized to elicit the same sweetness intensities as the original stimulus distributions. In these distributions, the mixtures were partly (distributions PosHalf and NegHalf) or totally (distributions PosUnm and NegUnm) replaced by equisweet unmixed fructose solutions. Stimuli were operationally defined to be equisweet in a specific experimental setting if they were expected to obtain the same mean sweetness responses in that setting (see intermediate analyses section). For the positively skewed and the negatively skewed stimulus distributions, two separate series of equisweet fructose solutions were obtained (labeled $\mathrm{K}-\mathrm{R}$ and $\mathrm{K}^{\prime}-\mathrm{R}^{\prime}$, respectively, in Table 2).

The six stimulus distributions shown in Table 2 were used to obtain eight different experimental conditions (Table 3 ). All subjects received either positively or negatively skewed stimulus distributions during the entire experiment to avoid between-session transfer of skew effects (Schifferstein, 1994b). The subjects who participated in two sessions all received a stimulus distribution with the maximum number of mixtures $(67 \%)$ in the first week. Seven days later, they were randomly assigned to a group evaluating a stimulus distribution with $67 \%$ mixtures (Mix, identical to Week 1), 27\% mixtures (Half), or no mixtures (Unm). Two groups
Table 3

The Eight Conditions Used in Experiment 1

\begin{tabular}{ccll}
\hline & & \multicolumn{2}{c}{ Distribution } \\
\cline { 3 - 4 } Condition & $n$ & Week 1 & Week 2 \\
\hline PM & 12 & PosMix & PosMix \\
PH & 12 & PosMix & PosHalf \\
PHc & 12 & PosHalfc & \\
PU & 12 & PosMix & PosUnm \\
NM & 12 & NegMix & NegMix \\
NH & 12 & NegMix & NegHalf \\
NHc & 12 & NegHalfc & \\
NU & 12 & NegMix & NegUnm \\
\hline
\end{tabular}

of subjects participated in one session only. These subjects received a Half distribution (conditions $\mathrm{PHc}$ and $\mathrm{NHc}$ ). These conditions were control conditions for the $\mathrm{PH}$ and $\mathrm{NH}$ conditions, respectively, and enabled an investigation to be made of transfer effects from the evaluation of a Mix distribution in Week 1 to the judgments of a Half distribution in Week 2.

Procedure. The subjects were instructed to judge the intensity of the perceived sweetness. The instructions emphasized that only the sweetness intensity was to be judged. Hedonic value and side tastes of the stimuli were to be disregarded. In the instructions, minimum sweetness and maximum sweetness were defined as equal to the sweetness intensities of the two stimuli of the reference pair (i.e., water and $2.25 \mathrm{M}$ fructose, respectively). The references were tasted at the beginning of the session and again after the 30th stimulus.

During the experimental session, the 30 stimuli from one stimulus distribution were evaluated twice. Responses were recorded on $150-\mathrm{mm}$ visual analog scales. The left and right ends of the response scales were labeled "not sweet at all" and "extremely sweet."

The subjects were requested to rinse their mouths thoroughly with demineralized water after each stimulus. The stimuli were presented at room temperature $\left(\sim 20^{\circ} \mathrm{C}\right)$ in polystyrene medicine cups. Each cup contained about $10 \mathrm{ml}$ of solution. The time interval between stimuli was $50 \mathrm{sec}$. The order in which the $30 \mathrm{stimuli}$ of each half session were presented was randomized, and differed between and within subjects.

Intermediate analyses. The results obtained with the "Mix" distributions in the first week were used to calculate the unmixed fructose concentrations expected to obtain sweetness responses equal to those of the eight fructose/citric acid mixtures. The functional relationship between fructose concentration and sweetness response was approximated by estimating a third-order polynomial with the sweetness response as the dependent variable $(y)$ and the natural logarithm of the fructose concentration as the independent variable $(x)$. For the positively skewed distribution, the function obtained was $y=125.7+32.3 x-18.5 x^{2}-4.9 x^{3}$ $\left(R^{2}=.997\right)$; that for the negative skew was $y=92.2+50.4 x-$ $6.4 x^{2}-5.7 x^{3}\left(R^{2}=.999\right)$. The equisweet fructose concentrations were then obtained by substituting each mixture sweetness rating for $y$ and solving for $x$.

Table 2

Frequency of Presentation of Each Stimulus in the Distributions Used in Experiment 1

\begin{tabular}{|c|c|c|c|c|c|c|c|c|c|c|c|c|c|c|c|c|c|c|c|c|c|c|c|c|c|c|c|c|c|c|c|c|c|c|}
\hline \multirow[b]{2}{*}{ Distribution } & \multirow[b]{2}{*}{ Water } & \multicolumn{9}{|c|}{ Unmixed Fructose } & \multicolumn{8}{|c|}{ Mixtures } & \multicolumn{8}{|c|}{ Matched Stimuli (Pos) } & \multicolumn{8}{|c|}{ Matched Stimuli (Neg) } \\
\hline & & 1 & 2 & 3 & 45 & 5 & 6 & 7 & 8 & 9 & A & $\mathrm{B}$ & $\mathrm{C}$ & D & $\mathrm{E}$ & $\mathrm{F}$ & $\mathrm{G}$ & $\mathrm{H}$ & $\mathrm{K}$ & $\mathrm{L}$ & $\mathrm{M}$ & $\mathrm{N}$ & $\mathrm{O}$ & $\mathrm{P}$ & $\mathrm{Q}$ & $\mathbf{R}$ & $\mathbf{K}^{\prime}$ & $L^{\prime}$ & $\mathbf{M}^{\prime}$ & $\mathrm{N}^{\prime}$ & $\mathrm{O}^{\prime}$ & $\mathbf{P}^{\prime}$ & $Q^{\prime}$ & $\mathrm{R}^{\prime}$ \\
\hline PosMix & 1 & 1 & 1 & 1 & 11 & 1 & 1 & 1 & 1 & 1 & 7 & 4 & 3 & 2 & 1 & 1 & 1 & 1 & 0 & 0 & 0 & 0 & 0 & 0 & 0 & 0 & 0 & 0 & 0 & 0 & 0 & 0 & 0 & 0 \\
\hline PosHalf & 1 & 1 & 1 & 1 & 11 & 1 & 1 & 1 & 1 & 1 & 1 & 1 & 1 & 1 & 1 & 1 & 1 & 1 & 6 & 3 & 2 & 1 & 0 & 0 & 0 & 0 & 0 & 0 & 0 & 0 & 0 & 0 & 0 & 0 \\
\hline PosUnm & 1 & 1 & 1 & 1 & 11 & 1 & 1 & 1 & 1 & 1 & 0 & 0 & 0 & 0 & 0 & 0 & 0 & 0 & 7 & 4 & 3 & 2 & 1 & 1 & 1 & 1 & 0 & 0 & 0 & 0 & 0 & 0 & 0 & 0 \\
\hline NegMix & 1 & 1 & 1 & 1 & 11 & 1 & 1 & 1 & 1 & 1 & 1 & 1 & 1 & 1 & 2 & 3 & 4 & 7 & 0 & 0 & 0 & 0 & 0 & 0 & 0 & 0 & 0 & 0 & 0 & 0 & 0 & 0 & 0 & 0 \\
\hline NegHalf & 1 & 1 & 1 & 1 & 11 & 1 & 1 & 1 & 1 & 1 & 1 & 1 & 1 & 1 & 1 & 1 & 1 & 1 & 0 & 0 & 0 & 0 & 0 & 0 & 0 & 0 & 0 & 0 & 0 & 0 & 1 & 2 & 3 & 6 \\
\hline NegUnm & 1 & 1 & 1 & 1 & 11 & 1 & 1 & 1 & 1 & 1 & 0 & 0 & 0 & 0 & 0 & 0 & 0 & 0 & 0 & 0 & 0 & 0 & 0 & 0 & 0 & 0 & 1 & 1 & 1 & 1 & 2 & 3 & 4 & 7 \\
\hline
\end{tabular}

Note-For explanation, see text. 
Statistical testing. If sweetness ratings for mixtures and unmixed substances are differentially affected by an experimental variable, ratings for unmixed stimuli may change in the direction opposite to the shift in mixture ratings. The test most sensitive for detecting differential context effects is, therefore, a test on changes in stimulus matches. To permit an analysis of variance (ANOVA) on stimulus matches, matching fructose concentrations were calculated using a matching-by-scaling procedure. This procedure is displayed graphically in Figure 2 for the responses to Mixture $F$ ( $7.1 \mathrm{mM}$ citric acid and $0.7 \mathrm{M}$ fructose) in the second session employing the PosMix distribution.

First, for each condition the relationship between the concentration of unmixed fructose and mean sweetness response (on a group level) was approximated by a second-order polynomial with the mean response as the dependent variable $(y)$ and the natural logarithm of the fructose concentration as the independent variable $(x)$. For the NegMix and the PosMix distributions of the first week, both functions had an $R^{2}$ value of 993 (data from 36 subjects). For the other distributions, the $R^{2}$ value ranged from .975 to .996 (data from 12 subjects). Subsequently, the mean individual response for each mixture was substituted for $y$, and the equation was solved for $x$ (pathway $\mathrm{A} \rightarrow \mathrm{B} \rightarrow \mathrm{C}$ in Figure 2). In this way, individual estimates for the concentration of fructose, equisweet to a specific mixture, were obtained. The natural logarithms of these matched fructose concentrations were then used in the ANOVA.

For $7 \%$ of the data in the second week of the PM ( 7 observations) and NM ( 6 observations) conditions, this procedure yielded impossible outcomes. The mean individual responses were either below the minimum or above the maximum of the estimated poly-

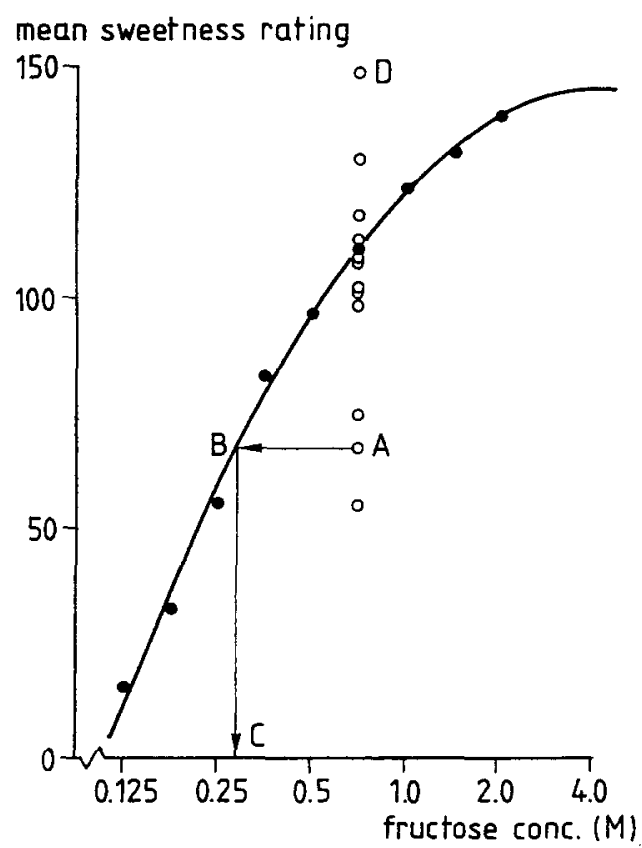

Figure 2. Explanation of the matching procedure, using data of the PosMix condition in the second week. The solid circles give the group mean responses to the unmixed fructose solutions. The curved line is the estimated group psychophysical function for the sweetness of fructose. The open circles give the mean individual ratings for Mixture $F$ (7.1 mM citric acid and $0.7 \mathrm{M}$ fructose). For each individual's mean rating $(A)$, the equisweet fructose concentration is determined by calculating the concentration of unmixed fructose corresponding to this response $(C)$. If the individual mixture response is higher than the maximum or lower than the minimum of the group psychophysical function (D), the corresponding fructose concentration has to be approximated in a different way (see text). nomial (Point D in Figure 2). For these data, the response was replaced by the closest response yielding a possible outcome.

\section{Results}

Mixture interactions. Figure 3 shows the sweetness responses to the unmixed fructose solutions and the fructose/citric acid mixtures for all stimulus distributions. Three-way (fructose level $\times$ acid percentage $\times$ subject) repeated measures ANOVAs of the responses to mixtures and to unmixed stimuli with the same fructose level were performed for each condition and each type of skew. Significant acid main effects were obtained for the two PosMix distributions $[F(2,70)=6.45$ and $F(2,22)=8.76$, $p<.01]$. Significant acid $\times$ fructose interactions were obtained in 5 out of 8 tests $[F(6,210)=4.26$ and $F(6,66) \geq$ $3.11, p<.01]$.

At the $2.0 \mathrm{M}$ fructose level, the sweetness ratings for the mixtures are lower than the ratings for unmixed fructose, indicating mixture suppression. A comparison of mean ratings yielded significant differences for 15 out of 16 tests (two-tailed $t$ tests, ${ }^{2} p<.05$ ). For the lower fructose levels, none of the negatively skewed distributions show significant suppression. In the positively skewed distributions, 7 of the 24 tests were significant $(p<.05)$. At low fructose levels, the mixture ratings exceed the rating for unmixed fructose in some cases. Only one of these increments is significant-that in the NegHalfc condition (Figure 3B) $(p<.05)$.

Positive versus negative skew. The degree of vertical separation between curves with solid circles and those with open circles demonstrates the size of the context effect for each condition and each stimulus type. In every panel, a context effect is evident: A positively skewed stimulus distribution yields higher sweetness ratings than does a negatively skewed distribution. For each stimulus type (unmixed or mixed) and each condition, the significance of the context effect was investigated in a three-way (skew $\times$ stimulus $\times$ subject) repeated measures ANOVA. In this analysis, skew was a between-subjects factor and stimulus was a withinsubjects factor. For the responses to unmixed fructose, the skew main effect $[F(1,70)=88.1$ and $F(1,22) \geq$ $10.7, p<.01]$ and the skew $\times$ stimulus interaction $[F(3,560)=6.7$ and $F(3,176) \geq 3.5, p<.01]$ were significant in each condition. In general, the skew main effect was also significant for the mixtures $[F(1,70)=28.1$ and $F(1,22) \geq 4.9, p<.05$ ], although it was of borderline significance in Figure $3 \mathrm{~B}[F(1,22)=2.8, p=.11]$. The skew $\times$ stimulus interaction was significant for the mixtures in Figures $3 \mathrm{~A}$ and $3 \mathrm{~B}[F(7,490)=6.43$ and $F(7,154)=2.69, p<.05]$, but not for those in Figures $3 \mathrm{C}$ and $3 \mathrm{D}[F(7,154) \leq 1.4, p>.20]$.

These analyses demonstrate that sweetness ratings for mixed and unmixed stimuli are affected by the type of skew in the stimulus distribution. In some cases, the size of the contextual shift is stimulus dependent. The next question to be answered concerns whether the type of skew affects both types of judgments to the same degree. This differential context effect is assessed by investigat- 

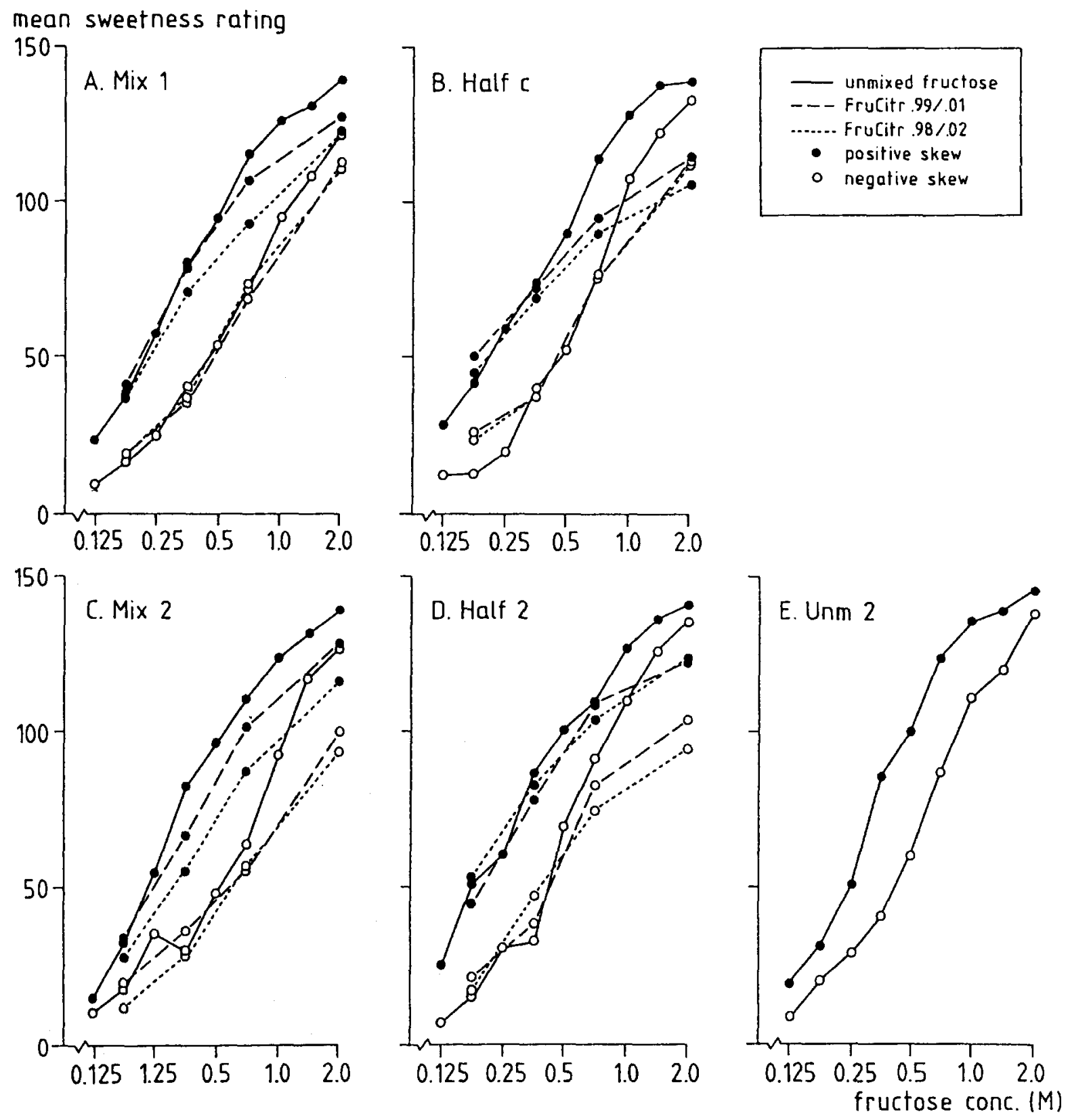

Figure 3. Mean sweetness ratings for unmixed fructose and the fructose/citric acid mixtures in positively and negatively skewed contexts. Panel $A$ shows the results of the PosMix-NegMix comparison for naive subjects $(n=36)$. Panel B shows the PosHalfc-NegHalfc comparison $(n=12$ naive subjects). Panel $C$ shows the PosMix-NegMix comparison for subjects at their second session $(n=12)$. Panel $D$ shows the PosHalf-NegHalf data for subjects at their second session $(n=12)$. Panel E shows the results of the PosUnm-NegUnm comparison $(n=12)$.

ing the fructose concentrations that matched the mixtures in rated sweetness intensity in each condition. For each of the panels A-D in Figure 3, a three-way (skew $X$ stimulus $\times$ subject) ANOVA was performed, using logtransformed fructose concentrations as input.

Figure 4 shows the fructose matches for the eight fructose/citric acid mixtures in the PosMix and the NegMix distributions. The data for naive subjects (Figure 4A) show that the matched concentrations in the negatively skewed context tend to be higher than those in the positively skewed context. The difference in matching concentrations seems to be largest for the mixtures with the lowest and the highest fructose concentrations. An ANOVA showed a significant skew $\times$ stimulus interaction $[F(7,490)=2.2, p=.035]$, but no main effect of skew $[F(1,70)=0.0, p=.87]$. For the NegMix and PosMix distributions in the second week (Figure 4B), both curves are similar and approximately monotonic. Neither the skew main effect $[F(1,22)=0.5, p=.50]$ nor the skew $\times$ stimulus interaction $[F(7,154)=0.3, p=.94]$ 


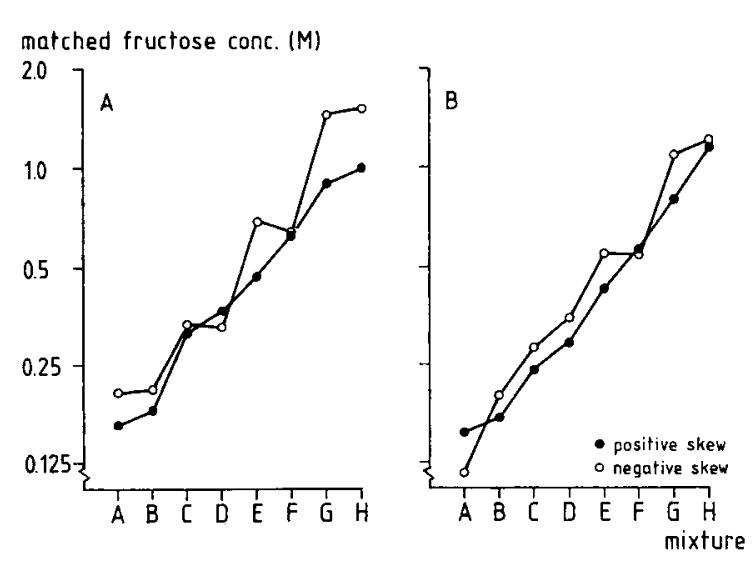

Figure 4. Matched fructose concentrations in the PosMix and NegMix conditions. Panel A gives the results for naive subjects $(n=36$ ). Panel B gives the results for subjects at their second session $(n=12)$.

was significant. The PosHalf-NegHalf comparisons yielded no significant skew effects or skew $\times$ stimulus interactions on fructose matches $(p>.15)$.

Carry-over effects. Panels A and B in Figure 3 show results for naive subjects. Panels $C, D$, and $E$ show results for subjects who had already participated in one Mix session. For each type of stimulus distribution, the results of the upper panel were compared with the results of the lower panel in a three-way (week $X$ stimulus $X$ subjects) repeated measures ANOVA. The week main effect appeared significant for the mixture ratings $[F(1,11)=$ $17.4, p<.01]$ and stimulus matches $[F(1,11)=16.9, p<$ $.01]$ in the NegMix condition only. This result is not surprising, since the previous paragraph reported a differential context effect to be significant in Week 1 and to be absent in Week 2 .

The number of mixtures. The effect of the differences between experimental conditions, varying with regard to the number of mixtures in the stimulus distribution, was investigated using three-way between- or within-subjects ANOVAs of sweetness responses and stimulus matches. None of the analyses provided a significant condition main effect $[F(1,22) \leq 4.6, p>.05]$. Out of 24 tests, 6 yielded a significant condition $\times$ stimulus interaction $[F(8,176) \geq 2.1$ for unmixed fructose and $F(7,154) \geq 2.2$ for mixtures and matches, $p<.05$ ].

Figure 5 shows the effect of the number of mixtures in the stimulus set upon the degree of sweetness suppression. In Mix distributions, $67 \%$ of the stimuli were mixtures; in Half distributions, $27 \%$ were mixtures. In general, the data lie in the vicinity of the diagonal. At low or negative degrees of suppression the points lie above the diagonal; at high levels of suppression they tend to lie below the diagonal. Estimation of a regression line through the 32 data points by minimizing the squared orthogonal distances from the line (Hampton, 1983; Williams, 1986) yielded $y=3.01+0.73 x$. Since the standard error of the slope equaled 0.11 , the slope differed significantly from $1.0(p<.05)$. This outcome suggests that the number of mixtures in the stimulus set affects the size and nature of the mixture interaction found in the responses.

In summary, Experiment 1 showed a substantial difference between mean ratings obtained in negatively skewed and positively skewed stimulus distributions. Contextual shifts in mixture ratings were approximately the same as the shifts in ratings for unmixed stimuli. In addition, replacing mixtures by equisweet, unmixed stimuli hardly affected responses. The relative number of mixtures in the stimulus set had a small, but systematic, effect on the degree of mixture interaction.

\section{EXPERIMENT 2}

Experiment 1 employed skewed and unskewed stimulus sets. In Experiment 2, a skewed set of mixtures was combined with a skewed set of unmixed stimuli. Subjects, stimuli, and experimental procedure were similar; only specific differences are mentioned below.

\section{Method}

Subjects. Forty-eight naive volunteers, 11 men and 37 women, ranging in age from 18 to 25 years (median age 20), participated in the experiment.

Stimuli. Eight fructose concentrations were used, ranging from 0.177 to $2.0 \mathrm{M}$ in equal log steps. The eight fructose/citric acid mixtures formed a FruCitr.98/.02 equiratio-mixture series. The fructose concentrations in the mixture series were equal to the fructose concentrations of the unmixed fructose solutions. The citric acid concentration ranged from 3.5 to $40 \mathrm{mM}$.

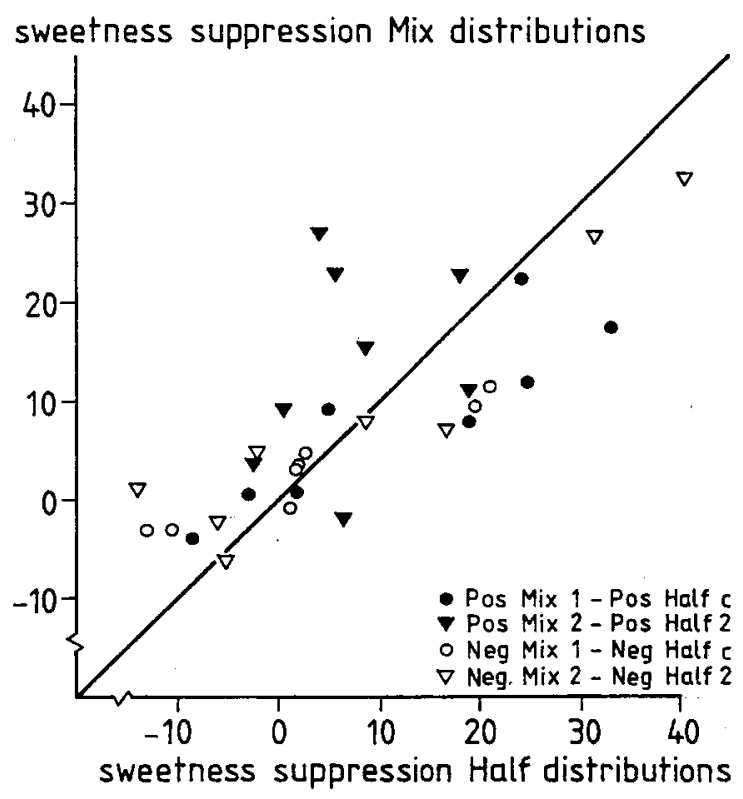

Figure 5. The relationship between the degrees of sweetness suppression in fructose/citric acid mixtures for stimuli presented in a stimulus set containing $27 \%$ mixtures (horizontal axis) and for those in a stimulus set containing $67 \%$ mixtures (vertical axis). The suppression found for the Half distributions of Experiment 1 is compared with suppression in the Mix distributions of Experiment 1. For each mixture, the degnee of sweetness suppression was calculated as the difference in sweetness rating between the unmixed fructose concentration and the mixture. 
Design. The two series of experimental stimuli were used to obtain differently skewed stimulus distributions. Four different conditions were obtained by combining one mixture distribution with one unmixed stimulus distribution (Table 4). Each subject evaluated all stimuli from one pair of stimulus distributions twice during a 1 -h session.

Statistical testing. As in Experiment 1, unmixed fructose concentrations were calculated that were expected to evoke the same sweetness responses as the eight fructose/citric acid mixtures. To permit an ANOVA on stimulus matches, the relationship between the concentration of unmixed fructose and the mean sweetness response (on a group level) was approximated. For each condition, a second-order polynomial was estimated with the mean response as the dependent variable $(y)$ and the natural logarithm of the fructose concentration as the independent variable $(x)$. The $R^{2}$ value of these regression equations ranged from .975 to .999 (data from 12 subjects).

For $26 \%$ ( 25 observations) of the responses in the PosPos condition, the regression equation yielded an impossible outcome: The maximum of this regression function occurred at $2.07 \mathrm{M}$, and many individual responses were higher than the corresponding response level (132.1). To select one fructose concentration, $C$, to match the responses above 132.1 , the sensitivity of the mean group response to changes in $C$ was determined. The mean group response was obtained by substituting the group mean of the individual matching concentrations for $x$ in the equation for the group psychophysical function, and solving for $y$. For $2.0<C<3.0$, all mean group responses obtained in this way were higher than the mean responses calculated from averaging the responses. Increasing $C$ from 2.0 to $3.0 \mathrm{M}$ resulted in an average increase in the responses for 5 mixtures with 2.2 units. Mixture ratings for three stimuli were unaffected by changes in $C$. Since the changes in responses are minimal, the matching fructose concentration $C$ was arbitrarily set to $2.5 \mathrm{M}$.

Since it does not seem appropriate to base conclusions on a data set in which $26 \%$ of the data were arbitrarily substituted, a supplementary analysis was performed. In an ANOVA of the sweetness responses, the skew $\times$ stimulus type interaction was tested against the stimulus type $\times$ subjects interaction for significance. This test investigated whether the sweetness ratings for mixed and unmixed stimuli (stimulus type) were differentially affected by stimulus context. The analysis assumes that the mixtures are approximately equisweet to the unmixed fructose concentrations used.

\section{Results}

Mixture interactions. To test the differential context effect using the raw data (Column 6 in Table 5), the mixtures and unmixed stimuli have to be approximately equisweet. For two out of four conditions, the mean sweetness ratings for mixed and unmixed stimuli did not differ significantly in an overall $F(1,22)$ test (PosPos, $p=$ .67 ; NegPos, $p=.42)$. In the PosNeg $(p=.06)$ and the

Table 4

The Four Conditions of Experiment 2 With Their Stimulus Frequency Distributions

\begin{tabular}{|c|c|c|c|c|c|c|c|c|c|c|c|c|c|c|c|c|c|}
\hline \multirow[b]{2}{*}{ Condition } & \multirow[b]{2}{*}{$n$} & \multicolumn{8}{|c|}{ Unmixed Stimuli } & \multicolumn{8}{|c|}{ Mixed Stimuli } \\
\hline & & $\overline{1}$ & 2 & 3 & 4 & 5 & 6 & 7 & $\overline{8}$ & $\overline{\mathrm{A}}$ & B & $\mathrm{C}$ & $\mathrm{D}$ & E & $\mathrm{F}$ & $G$ & $\mathrm{H}$ \\
\hline PosPos & 12 & 5 & 3 & 2 & 1 & 1 & 1 & 1 & 1 & 5 & 3 & 2 & 1 & 1 & 1 & 1 & 1 \\
\hline PosNeg & 12 & 5 & 3 & 2 & 1 & 1 & 1 & 1 & 1 & 1 & 1 & 1 & 1 & 1 & 2 & 3 & 5 \\
\hline NegPos & 12 & 1 & 1 & 1 & 1 & 1 & 2 & 3 & 5 & 5 & 3 & 2 & 1 & 1 & 1 & 1 & 1 \\
\hline NegNeg & 12 & 1 & 1 & 1 & 1 & 1 & 2 & 3 & 5 & 1 & 1 & 1 & 1 & 1 & 2 & 3 & 5 \\
\hline
\end{tabular}

Note-Perceived sweetness intensity increases from left to right in both stimulus series.
Table 5

$F$ values Obtained in ANOVA Making

Paired Comparisons of the Four Experimental Conditions of Experiment 2

\begin{tabular}{ccccc}
\hline & & & \multicolumn{2}{c}{ Differential Effect } \\
\cline { 4 - 5 } Conditions & Unmixed & Mixtures & Matches & Direct Test \\
\hline PosPos PosNeg & $7.5 \dagger$ & $14.6 \ddagger$ & $4.7^{\dagger} \dagger$ & $3.4^{*}$ \\
PosPos NegPos & $9.0 \ddagger$ & $9.0 \ddagger$ & 1.4 & 1.2 \\
PosPos NegNeg & $10.2 \ddagger$ & $19.5 \ddagger$ & $6.8 \dagger$ & $5.4 \dagger$ \\
PosNeg NegPos & 0.1 & 0.8 & 1.0 & 1.0 \\
PosNeg NegNeg & 0.1 & 0.4 & 0.1 & 0.1 \\
NegPos NegNeg & 0.0 & 2.3 & 1.9 & 2.1 \\
\hline
\end{tabular}

Note-Columns 3 and 4 show the outcome for testing whether the ratings for the unmixed fructose solutions and the fructose/citric acid mixtures were significantly affected by context; Columns 5 and 6 show the result of testing whether the mixtures and the unmixed stimuli were differentially affected by context. For Column 5 , the test was performed on log-transformed fructose matches, while for Column 6 it was performed on the raw data. ${ }^{*} p<.10 ; \dagger p<.05 ; \ddagger p<.01$.

NegNeg $(p=.02)$ conditions, however, the mean ratings were lower for the mixtures. In both conditions, 4 of the 8 mixtures showed significant suppression (two-tailed $t$ tests, ${ }^{3} p<.05$ ). In the PosPos condition, the two mixtures with the lowest concentration levels showed significant enhancement $(p<.05)$. Since the stimuli used in each condition are the same and the interactions found are not consistent over conditions, these results show that the mixed and unmixed stimuli are similar enough to allow an analysis of the differential context effect using raw data.

Contextual effects on sweetness ratings and stimulus matches. Figure 6 shows the mean sweetness ratings for unmixed (Panel A) and mixed (Panel B) stimuli in the PosPos, PosNeg, NegPos, and NegNeg conditions. Stimulus distribution effects were tested for statistical significance in three-way (skew $\times$ stimulus $\times$ subjects) between-subjects ANOVAs (Table 5).

For the unmixed fructose solutions, the PosPos curve is clearly separated from the other three curves, which are highly similar. Since the NegNeg condition is one of the extreme conditions, one would expect the NegNeg curve to be located below the PosNeg and the NegPos curves. However, the NegNeg curve crosses the two curves in the middle. For the fructose/citric acid mixtures, the ratings in the PosPos curve are considerably higher than the ratings in the other three conditions. The NegNeg condition appears to obtain the lowest responses.

Statistical analysis shows that significant skew main effects are found only for comparisons involving the PosPos condition. Apparently, the substitution of one or both positively skewed stimulus distributions with negatively skewed ones evokes shifts in responses to mixed and unmixed stimuli. Substituting one of the stimulus distributions in the NegNeg condition with a positively skewed distribution hardly affects the mean responses.

Testing for a skew main effect in the stimulus matches or a skew $\times$ stimulus type interaction in the raw data yielded similar outcomes: The differential effect is significant for the PosPos-PosNeg and the PosPos-NegNeg 
comparison. The contextual shift in responses was larger for mixed than for unmixed stimuli when either the mixture distribution or both distributions changed from positively to negatively skewed.

The relationship between response variation and size of the context effect. For Experiments 1 and 2, the relationship between the mean standard error of a response (averaged over standard errors for the positive and the negative skew) and the size of the context effect (the difference between the mean ratings in the positive context and the negative context) was determined for each condition. For the stimuli presented in a skewed stimulus distribution, the standard errors were based on two responses from each subject.

The standard error is higher for the mixture ratings than for the ratings of unmixed stimuli (Table 6). The data for unmixed stimuli show a positive correlation between response variation and the size of the context effect. Even in Experiment 2, where the context effect is small, the two variables are positively correlated. For mixtures, however, the two variables are not associated $(p>.14)$.

\section{Discussion}

In the introduction three factors were hypothesized to affect the size of contextual effects, namely, stimulus complexity, response variability, and the degree of integration. These variables are discussed below.

Stimulus complexity. Increased stimulus complexity is hypothesized to increase the size of the contextual shifts-implying a differential context effect between

Table 6

The Relationship Between Response Variability and the Size of the Context Effect

\begin{tabular}{|c|c|c|c|c|c|}
\hline Condition & $n$ & Stimuli $^{\mathrm{a}}$ & Size $^{b}$ & $\mathrm{SE}^{\mathrm{c}}$ & $r^{\mathrm{d}}$ \\
\hline \multicolumn{6}{|c|}{ Unmixed stimuli } \\
\hline \multicolumn{6}{|l|}{ Experiment 1} \\
\hline Mix, 1st session & 36 & 10 & 26.5 & 2.75 & $0.93 \dagger$ \\
\hline Mix, 2nd session & 12 & 10 & 24.1 & 4.24 & $0.77 \dagger$ \\
\hline Half, control & 12 & 10 & 23.4 & 4.32 & $0.92 \dagger$ \\
\hline Half, 2nd session & 12 & 10 & 22.5 & 4.64 & $0.84 \dagger$ \\
\hline Unm, 2nd session & 12 & 10 & 21.2 & 4.01 & $0.92 \dagger$ \\
\hline Experiment 2 & 12 & 16 & 8.5 & 5.24 & $0.59^{*}$ \\
\hline \multicolumn{6}{|c|}{ Mixtures } \\
\hline \multicolumn{6}{|l|}{ Experiment 1} \\
\hline Mix, 1st session & 36 & 8 & 26.7 & 3.96 & -0.03 \\
\hline Mix, 2nd session & 12 & 8 & 26.0 & 6.34 & 0.28 \\
\hline Half, control & 12 & 8 & 17.6 & 7.71 & -0.24 \\
\hline Half, 2nd session & 12 & 8 & 26.5 & 7.70 & 0.30 \\
\hline Experiment 2 & 12 & 16 & 24.4 & 6.74 & -0.01 \\
\hline
\end{tabular}

${ }^{\mathrm{a}}$ The number of stimuli on which the comparison is based. ${ }^{\mathrm{b}} \mathrm{The}$ mean value of the size of the context effect (rating in positive context minus rating in negative context), averaged over stimuli. ' $T$ The standard error of the mean ratings, averaged over contexts and stimuli. For each subject, only two responses to each stimulus were used in order to create equal numbers of observations for each stimulus. ${ }^{d}$ The Pearson correlation coefficient for the association between size of the context effect and the standard error of the mean sweetness rating.

For Experiment 2, the PosNeg condition was paired with the PosPos condition, while the NegPos condition was paired with the NegNeg condition. This was an arbitrary choice. ${ }^{*} p<.05 ; \dagger p<.01$. mixed and unmixed stimuli-and to increase the degree of random variation.

As regards the size of the contextual shifts, Experiment 1 yielded no significant skew main effect for fructose matches, whereas Experiment 2 produced a significant skew effect in the PosPos-PosNeg and the PosPos-NegNeg comparisons. Similarly, Figure 6 and Table 6 show that the average size of the contextual shifts in Experiment 2 is 8.5 for unmixed stimuli and 24.4 for mixed stimuli. In Experiment 1, the shift sizes for mixed and unmixed stimuli are comparable. Hence, Experiment 2 indicates that increased stimulus complexity increases the size of the context effect, whereas Experiment 1 does not indicate this.

The discrepancies between Experiments 1 and 2 may be due to the fact that stimulus sets differed more extremely between conditions in Experiment 2. In Experiment 1 , the skewed distribution was combined with a uniform distribution. Experiment 2 showed that only a skewed distribution combined with a positively skewed distribution yielded a differential context effect (PosPosPosNeg). The PosPos-NegNeg comparison is even more extreme, since it involves changes in two skews. A skewed distribution combined with a negatively skewed one yielded no differential effect (NegPos-NegNeg and PosNeg-NegNeg).

As regards the standard errors of the responses, Table 6 suggests that mixture ratings are more variable than are ratings for unmixed fructose. Consequently, the present findings support the hypothesis that stimulus complexity increases the susceptibility to contextual shifts and the variation in the responses.

Response uncertainty. An elevated number of appropriate response alternatives is likely to increase the sensitivity to contextual manipulations. Contrary to the hypothesis, Table 6 shows that this relationship is evident for the responses to unmixed fructose, but is not evident for mixtures. Although response selection also occurs for mixture ratings, mixture ratings may suffer from the additional increment in variation in the internal representation. Consequently, the contextual bias in response selection may be overshadowed or counteracted by the complexity effect, whereas this effect is absent in ratings for unmixed stimuli.

The present findings concur with Ross and DiLollo's (1971) findings that response-scale effects can account for contextual effects for unidimensional stimuli. For multidimensional stimuli, however, they explained context effects by a judgmental model for the complex determination of heaviness judgments ignoring responsescale effects (Ross \& DiLollo, 1968). Others have also reported that contextual shifts observed for multidimensional stimulus sets are due to shifts in internal representations (Marks, 1992, 1993; Mellers \& Birnbaum, 1982; Schifferstein, 1994a; Schneider \& Parker, 1990), whereas changes in response-output function account for context effects obtained with unidimensional stimulus sets (Birnbaum, Parducci, \& Gifford, 1971; Marks, 1993; Mellers \& Birnbaum, 1982). 


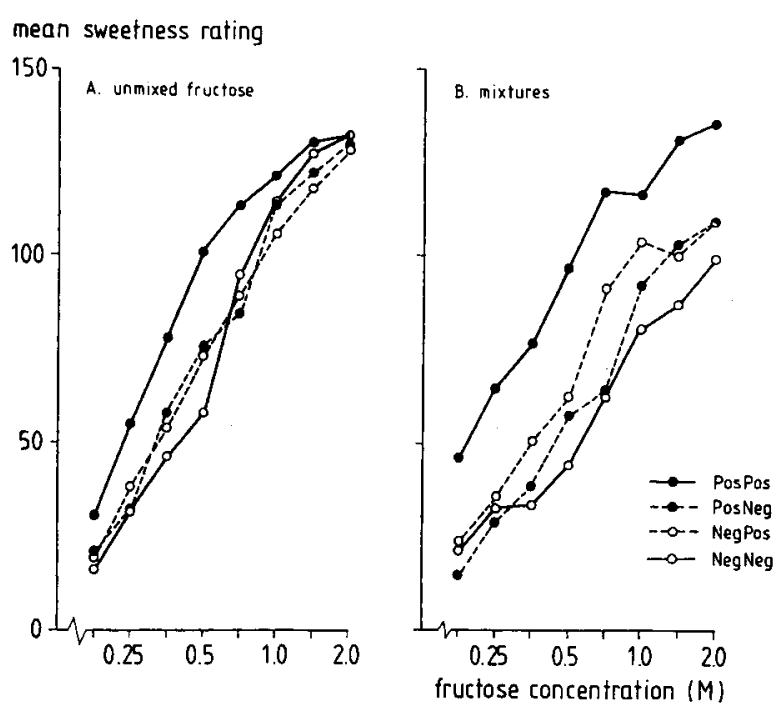

Figure 6. Mean sweetness ratings for unmixed fructose (panel A) and fructose/citric acid mixtures (panel B) in the four conditions of Experiment 2.

Degree of integration. For subjects who integrate all stimuli into one subjective stimulus context, stimulus matches will not shift with contextual manipulations. The present experiments evaluated whether stimulus matches depend on the relative number of mixtures in the stimulus set. None of the Mix-Half comparisons of Experiment 1 showed a significant condition main effect in ANOVA of the stimulus matches, nor did the NegPosPosNeg comparison of Experiment 2. Although Figure 5 suggests that the percentage of mixtures affects stimulus matches, the deviations from the diagonal are small and point in opposite directions for small and large degrees of suppression. For large degrees of suppression, the present findings concur with reports by Kroeze (1982) regarding saltiness suppression by sucrose, and by Schifferstein (1994b) regarding bitterness suppression by $\mathrm{NaCl}$. Qualitative stimulus characteristics could have more effect on stimulus ratings in studies finding more mixture suppression than the present one.

The previous paragraph suggests that subjects integrate the "unmixed" context with the "mixed" context when they judge sweetness intensity. However, some outcomes suggest that the integration is not complete: The PosPos-PosNeg comparison yields a significantly larger contextual shift for the mixtures than for the unmixed stimuli, whereas the PosPos-NegPos comparison produces no differential effect. Apparently, a change in mixture distribution is more effective in producing a shift in the mixture ratings than is a change in the unmixed fructose distribution. Such a differential effect was more likely to occur in Experiment 2 than in Experiment 1 , because the mixture acid levels were higher and consequently the differences between stimulus sets were larger in Experiment 2.

Conclusion. Sweetness intensity judgments of fructose and fructose/citric acid mixtures are hardly affected by qualitative changes of stimulus set composition (percentage of mixtures), pointing to a high degree of psychological integration of the two stimulus types. Substantial differences in the quantitative characteristics of the stimulus sets (type of skew) have indicated that the mixture ratings may be more susceptible to contextual shifts than are ratings for unmixed fructose. Analyses indicate that context mainly affects internal representation in mixture processing and affects response selection in the processing of unmixed fructose. Although the impact of experimental stimulus context in the present study was limited, it remains an interesting variable in future studies on mixture perception.

\section{REFERENCES}

Birnbaum, M. H., Parducci, A., \& Gifford, R. K. (1971). Contextual effects in information integration. Journal of Experimental Psychology, 88, 158-170.

Borg, G., Diamant, H., OAKley, B., Ström, L., \& Zotterman, Y. (1967). A comparative study of neural and psychophysical responses to gustatory stimuli. In T. Hayashi (Ed.), Olfaction and taste II (pp. 253-264). Oxford: Pergamon Press.

DE GraAF, C., \& FriJTERS, J. E. R. (1987). Concentrations of $\mathrm{NaCl}$ and sucrose: Equality in perceived taste intensity. Chemical Senses, 12 , 631-635.

De GraAF, C., \& Frijters, J. E. R. (1989). Interrelationships among sweetness, saltiness and total taste intensity of sucrose, $\mathrm{NaCl}$ and sucrose $/ \mathrm{NaCl}$ mixtures. Chemical Senses, 14, 81-102.

ERICKSON, R. P. (1982). Studies on the perception of taste: Do primaries exist? Physiology \& Behavior, 28, 57-62.

ERICKSON, R. P., \& COVEY, E. (1980). On the singularity of taste sensations: What is a taste primary? Physiology \& Behavior, 25, $527-$ 533.

Frijters, J.E. R. (1993). Functional measurement in the study of mixture percepts. Chemical Senses, 18, 93-100.

Fritters, J. E. R., \& OUdE Ophuis, P. A. M. (1983). The construction and prediction of psychophysical power functions for the sweetness of equiratio sugar mixtures. Perception, 12, 753-767.

HAMPTON, J. A. (1983). A note on describing the linear relation between a pair of correlated dependent variables. Bulletin of the British Psychological Society, 36, 408-409.

HaUbensaK, G. (1992). The consistency model: A process model for absolute judgments. Journal of Experimental Psychology: Human Perception \& Performance, 18, 303-309.

HeLson, H. (1964). Adaptation level theory. New York: Harper \& Row. KROEZE, J. H. A. (1982). The influence of relative frequencies of pure and mixed stimuli on mixture suppression in taste. Perception \& Psychophysics, 31, 276-278.

KROEZE, J. H. A. (1983). Successive contrast cannot explain suppression release after repetitious exposure to one of the components of a taste mixture. Chemical Senses, 8, 211-223.

KROEZE, J. H. A., \& BARTOSHUK, L. M. (1985). Bitterness suppression as revealed by split-tongue taste stimulation in humans. Physiology \& Behavior, 35, 779-783.

MARKs, L. E. (1982). Psychophysical measurement: Procedures, tasks, scales. In B. Wegener (Ed.), Social attitudes and psychophysical measurement (pp. 43-71). Hillsdale, NJ: Erlbaum.

MARKs, L. E. (1989). The dynamics of ratio scaling. In S. J. Bolanowski \& G. A. Gescheider (Eds.), Ratio scaling of psychological magnitude. Papers in honor of S.S. Stevens (pp. 27-42). Hillsdale, NJ: Erlbaum.

MARKs, L. E. (1992). The contingency of perceptual processing: Context modifies equal-loudness relations. Psychological Science, 3, 285-291.

Marks, L. E. (1993). Contextual processing of multidimensional and unidimensional auditory stimuli. Journal of Experimental Psychology: Human Perception \& Performance, 19, 227-249. 
Marks, L. E., \& WARner, E. (1991). Slippery context effect and critical bands. Journal of Experimental Psychology: Human Perception \& Performance, 17, 986-996.

MCBRide, R. L. (1985). Stimulus range influences intensity and hedonic ratings of flavor. Appetite, 6, 125-131.

Mellers, B. A., \& Birnbaum, M. H. (1982). Loci of contextual effects in judgment. Journal of Experimental Psychology: Human Perception \& Performance, 8, 582-601.

MONTGOMERY, H. (1982). Intra- and interindividual variations in the form of psychophysical scales. In B. Wegener (Ed.), Social attitudes and psychophysical measurement (pp. 339-350). Hillsdale, $\mathrm{NJ}$ : Erlbaum.

O'Mahony, M., Atassi-Sheldon, S., Rothman, L., \& MurphyEllison, T. (1983). Relative singularity/mixedness judgements for selected taste stimuli. Physiology \& Behavior, 31, 749-755.

PANGBorn, R. M. (1960). Taste interrelationships. Food Research, 25, 245-256.

Parducci, A. (1965). Category judgment: A range-frequency model. Psychological Review, 72, 407-418.

ParducCi, A. (1974). Contextual effects: A range-frequency analysis. In E. C. Carterette \& M. P. Friedman (Eds.), Handbook of perception: Vol. II. Psychophysical judgment and measurement (pp. 127141). New York: Academic Press.

Parducci, A., Knobel, S., \& Thomas, C. (1976). Independent contexts for category ratings: A range-frequency analysis. Perception \& Psychophysics, 20, 360-366.

ParducCI, A., \& Wedell, D. H. (1986). The category effect with rating scales: Number of categories, number of stimuli, and method of presentation. Journal of Experimental Psychology: Human Perception \& Performance, 12, 496-516.

RANKIN, K. M., \& MARKS, L. E. (1991). Differential context effects in taste perception. Chemical Senses, 16, 617-629.

RISKEY, D. R. (1982). Effects of context and interstimulus procedures in judgments of saltiness and pleasantness. In J. T. Kuznicki, R. A. Johnson, \& A. F. Rutkiewic (Eds.), Selected sensory methods: Problems and applications to measuring hedonics (pp. 71-83). Philadelphia, PA: American Society for Testing and Materials.

RISKEY, D. R., \& DESOR, J. (1980). Independence and nonindependence between taste contexts. Unpublished manuscript, General Foods Corporation Technical Center, White Plains, NY.

Riskey, D. R., ParducCI, A., \& BEAUChamp, G. K. (1979). Effects of context in judgments of sweetness and pleasantness. Perception \& Psychophysics, 26,171-176.

Ross, J., \& DiLollo, V. (1968). A vector model for psychophysical judgment. Journal of Experimental Psychology Monographs, 77 $(3,2), 1-16$.

Ross, J., \& DiLollo, V. (1971). Judgment and response in magnitude estimation. Psychological Review, 78, 515-527.

Satterthwaite, F. W. (1946). An approximate distribution of estimates of variance components. Biometrics Bulletin, 2, 110-114.
SChifferstein, H. N. J. (1994a). Contextual effects in difference judgments. Manuscript submitted for publication.

SCHIFFERSTEIN, H. N. J. (1994b). Contextual effects in the perception of quinine $\mathrm{HCl} / \mathrm{NaCl}$ mixtures. Chemical Senses, 19, 113-123.

SCHIfFERSTEIN, H. N. J., \& FriJTERS, J. E. R. (1992a). Contextual and sequential effects on judgments of sweetness intensity. Perception \& Psychophysics, 52, 243-255.

SChiffERStein, H. N. J., \& FriJTERs, J. E. R. (1992b). Sweetness does not habituate during a sip-and-spit experiment. Physiology \& Behavior, 51, 331-336.

SChNeIder, B., \& Parker, S. (1990). Does stimulus context affect loudness or only loudness judgments? Perception \& Psychophysics, 48, 409-418.

Schutz, H. G., \& Pilgrim, F. J. (1957). Differential sensitivity in gustation. Journal of Experimental Psychology, 54, 41-48.

Stevens, J. C., \& Marks, L. E. (1980). Cross-modality matching functions generated by magnitude estimation. Perception \& Psychophysics, 27, 379-389.

VOLLMECKE, T. A. (1987). The influence of context on sweetness and pleasantness evaluation of beverages. Unpublished doctoral dissertation, University of Pennsylvania, Philadelphia.

WARD, L. M. (1979). Stimulus information and sequential dependencies in magnitude estimation and cross-modality matching. Journal of Experimental Psychology: Human Perception \& Performance, 5 , 444-459.

WILLIAMS, R. B. G. (1986). Intermediate statistics for geographers and earth scientists. London: Macmillan.

\section{NOTES}

1. Marks (1989) has argued that perception may change by cognitive factors through top-down processing. In that case, the first two stages of Figure 1 should not be regarded as a sequence, but as two processes that occur simultaneously. The main merit of the framework in Figure 1 is that it shows that scaling of perceived intensity involves both sensory and cognitive elements. Sensations unaffected by cognition cannot be assessed in psychophysical research because the execution of every task requires cognitive processing.

2. Due to unequal sample sizes and correction for unequal variances (Satterthwaite, 1946), the degrees of freedom of the $t$ tests varied from 84 to 574 (median 153) for the NegMix 1 and PosMixl conditions, and from 27 to 190 (median 46) for the other conditions. Note that 3.2 significant outcomes are expected to occur by chance with the present $\alpha$ level (.05) for the entire number of tests performed (64).

3. Due to differences in numbers of observations per stimulus and correction for unequal variances (Satterthwaite, 1946), the degrees of freedom for the $t$ tests varied from 25 to 223 , with a median value of 52

(Manuscript received August 26, 1992; revision accepted for publication February 15, 1994.) 